\title{
Haïti 2010 : les leçons d'une catastrophe
}

Jean Marie Théodat

\section{(QpenEdition \\ Journals}

\section{Édition électronique}

URL : http://journals.openedition.org/echogeo/11682

DOI : $10.4000 /$ echogeo. 11682

ISSN : 1963-1197

\section{Éditeur}

Pôle de recherche pour l'organisation et la diffusion de l'information géographique (CNRS UMR 8586)

\section{Référence électronique}

Jean Marie Théodat, « Haïti 2010 : les leçons d'une catastrophe », EchoGéo [En ligne], Sur le Vif, mis en ligne le 08 février 2010, consulté le 02 mai 2019. URL : http://journals.openedition.org/echogeo/11682 ; DOI : 10.4000/echogeo.11682

Ce document a été généré automatiquement le 2 mai 2019.

\section{(c) (i) ()}

EchoGéo est mis à disposition selon les termes de la licence Creative Commons Attribution - Pas d'Utilisation Commerciale - Pas de Modification 4.0 International 


\title{
Haïti 2010 : les leçons d'une catastrophe
}

\author{
Jean Marie Théodat
}

1 Le tremblement de terre qui a ravagé la capitale haïtienne et fait plus de 200000 morts, constitue un événement global, d'une importance majeure qui étonne une époque habituée à percevoir ce pays comme la scène où se répète une tragédie humaine écrite depuis plus de deux siècles. On ne comprendra toutes leçons du séisme que lorsque l'onde de choc provoquée dans les consciences par ce phénomène extraordinaire aura cessé ses effets émotionnels les plus vifs. L'on pourra réfléchir alors aux inflexions que la catastrophe révèle à plusieurs égards. Nous proposons une analyse à deux échelles pour considérer à la fois les glissements que ce séisme dénote dans les relations internationales, et ce qu'il dévoile des fondements de la nation haïtienne, née d'une révolution d'esclaves en 1804.

Dans les relations internationales d'abord, un singulier déplacement de lignes est à l'œuvre où il est permis de voir les éléments liminaires d'une nouvelle forme de l'exercice de la puissance : l'intervention humanitaire unilatérale, massive, édifiante. Un tel schéma est appelé à se répéter dans les années à venir compte tenu de la récurrence des catastrophes naturelles et de l'incapacité des pays les plus pauvres à faire face aux nouveaux défis posés par les bouleversements climatologiques en cours. Les Etats-Unis d'Amérique pour avoir dépêché en Haïti un bataillon de plus de 20000 hommes, parmi les plus entraînés de l'armée américaine et, pour les avoir fait se poser sur la pelouse du palais national, symbole de l'indépendance haïtienne, ont été critiqués, notamment en France, pour le caractère arrogant de leur attitude.

3 En fait, le gouvernement américain, soucieux de ne pas répéter la même erreur qu'à l'occasion du cyclone Katrina qui dévasta la Nouvelle-Orléans en 2005, réagit avec une promptitude et une détermination qui seraient parues normales s'il s'était agi de porter secours à des civils américains sur leur propre territoire. Le monde jugea la diligence américaine excessive, envahissante, donc suspecte. Malgré l'ampleur du désastre il y eut en Haïti même des voix pour y voir une nouvelle Occupation après celle de 1915. Signe que l'image négative laissée par les équipées américaines dans cette partie de la Caraïbe 
est encore vivace dans les esprits, et que même muni de brancards, le marine inspire la peur.

4 Cependant, velléités impérialistes ou pas de la part des Américains, la question reste posée de savoir à quelle autorité revient de porter secours, en toute légitimité, aux populations les plus démunies, touchées par des catastrophes en tous genres, lorsque des Etats faibles, fragiles ou faillis, se révèlent incapables d'organiser les secours. La planète entière assistera-t-elle, spectatrice passive, par médias interposés, à leur insupportable agonie?

5 Il est important de signaler que la République dominicaine, pays qui partage l'île avec Haïti, et avec lequel les relations n'ont pas toujours été harmonieuses, fut le premier pays à voler au secours des Haïtiens aux abois : les premiers soins aux victimes, la première visite d'un chef d'Etat étranger, sont venus de Santo Domingo. C'est l'occasion pour les deux pays de démontrer qu'au-delà des tensions habituelles entre les deux capitales, une insularité partagée tient les deux peuples solidaires, pour le meilleur et pour le pire.

Il apparaît ainsi que porter secours aux sinistrés est un attribut tacite de la puissance, qui distingue les plus grandes des autres nations. L'agacement non dissimulé de la France, empêchée de débarquer à Port-au-Prince toute à son aise, par la prise de contrôle de l'aéroport Toussaint Louverture par les Américains, illustre à l'envi l'ombrage que la présence américaine porte aux prétentions des autres puissances. Cet empressement subit des pays amis constitue un tournant dans la perception d'Haïti, vu jusqu'alors comme un trou noir dans la galaxie des relations internationales. Mais c'est aussi l'illustration du fait que l'intervention humanitaire, c'est l'occasion d'une mise en scène de la puissance avec d'autres moyens que les armes; moyens médicaux et médiatiques, certes, mais tout aussi efficaces à forger d'une nation une image de force et de confiance en elle-même.

7 A la différence des territoires ayant un intérêt géographique ou économique propre, qui leur confère un enjeu stratégique clair au point que des puissances sont disposées à recourir à la guerre pour s'y installer les premières et s'y maintenir, Haïti n'a rien qui attire pour sa valeur marchande. Le vide créé par l'absence d'un Etat structuré laisse la place à un substitut d'autorité, dont les pays étrangers prennent, par temps de crise, la place: depuis 2004 la force d'interposition de l'ONU, la MINUSTAH, d'environ 9000 hommes, assure la sécurité et la stabilisation économique et sociale d'Haïti. Mais, au quotidien, l'absence d'Etat se fait cruellement sentir. Banc d'essai d'une nouvelle forme de gouvernance internationale, le pays est pourtant assisté d'une escorte d'experts et de spécialistes qui depuis des années réfléchissent au moyen de le sortir de la pauvreté : plus de 800 ONG sont à l'œuvre dans le pays, avec chacune des responsabilités fragmentées qui donnent un sentiment d'atomisation du corps social. Cette politique aboutit souvent à la dilution des responsabilités dans une sorte de paralysie mutuelle des services dépendant d'instances de décisions distinctes, agissant de façon sinon toujours contradictoire, du moins souvent sans plan et sans concertation.

8 Parmi les grandes puissances, aucune n'a envie de s'y installer durablement, cependant toutes ont éprouvé le besoin de s'y faire représenter. C'est qu'au-delà de l'émotion planétaire suscitée par l'ampleur des dégâts et le nombre de victimes, se joue la capacité d'un pays à se projeter dans les représentations collectives comme une nation civilisée, généreuse et animée d'intentions pacifiques. Sans parler plus trivialement des espoirs de retombées post-catastrophe, en terme de contrat de reconstruction, d'équipement et de relance de l'économie. Si la Chine, le Brésil, le Venezuela, Cuba, et même la Corée du Nord 
ont tenu à manifester leur présence sur le sol d'Haïti, à seulement $900 \mathrm{~km}$ des côtes américaines, c'est par esprit de fraternité avec un peuple fier, sans doute, mais aussi, une façon spectaculaire de rappeler aux Américains qu'ils n'ont pas le monopole de la générosité. Néanmoins, cette crise est surtout l'occasion pour les Etats-Unis d'édifier le monde en exposant les attributs de la puissance et, accessoirement, de démontrer l'utilité civile du budget militaire considérablement gonflé d'un Etat en guerre

D'autres flexions, économiques et sociales, sont la conséquence d'une crise structurelle plus longue que l'aléa tectonique rend flagrante en rappelant la vulnérabilité globale de la nation haïtienne.

Celle-ci semble construite, comme la ville, sur une ligne de faille sociale par où s'affrontent, dès l'indépendance, deux logiques antagoniques. L'une persistant à vouloir réhabiliter les anciennes plantations et reprendre la production de façon à maintenir les revenus de la première colonie sucrière du Nouveau Monde. L'autre consistant en l'apprentissage de la liberté pour les descendants d'Africains libérés au prix d'une guerre qui fit plus de 100000 victimes : que signifie être libre, après avoir été esclave? Comment gagner sa vie sans aliéner sa liberté ?

11 La guerre d'indépendance qui dura de 1791 à 1803 avait causé d'importants dommages à l'économie. Les plantations, symboles du lien colonial honni par les insurgés, furent la cible de la vindicte populaire: les systèmes d'irrigation, les bâtiments industriels furent réduits en cendres et les terres des colons exterminés versées au domaine de l'Etat en 1804.

12 Cependant, après l'abolition de l'esclavage en 1793, il était entendu que le statut des anciens esclaves serait conditionné par une double astreinte : l'assignation au travail de la terre et le service militaire. Le statut de paysan, régi par les règlements de culture officiels, définit alors un être non libre de ses mouvements, et obligé de travailler pour le compte d'autrui. Salarié, mais assigné à résidence.

13 C'est contre cette double contrainte que se mit en place la nouvelle paysannerie haïtienne. Regroupés en hameaux familiaux, sur les ruines des anciennes plantations, les lakou furent pour les nouveaux libres l'espace d'une nouvelle expérience politique économique et sociale sans égale alors dans les Amériques. Ils surent mettre en place des stratégies collectives pour accéder à la terre et résister aux tentatives de réhabilitation de la grande plantation. Contre la nouvelle élite (formée des anciens libres de couleur et d'officiers de l'armée) d'abord, puis durant l'Occupation américaine de 1915 à 1934, les paysans haïtiens ont mis en place des stratégies de résistance, armée ou non violente, dont une agriculture que l'on peut caractériser de légitime défense. Celle qui a permis de faire face à la triple injonction de nourrir le pays, d'assurer sa défense et de payer l'indemnité d'indépendance à la France. Au prix de la belle ordonnance des anciennes plantations, des ateliers tournant à plein régime à la période de la roulaison, des parcelles irriguées tirées au cordeau, etc. les jardins paysans adoptèrent des méthodes plus extensives. Les structures agraires s'adaptèrent au nouveau statut des hommes: une nouvelle forme de marronnage se mit en place. Il n'y a donc pas de fatalité à la faillite de l'économie haïtienne : c'est la ruine annoncée d'un pays éprouvé par une crise politique grave et qui dut consacrer l'essentiel de ses ressources, non pas à reconstituer ses forces, mais à faire accepter son droit à l'existence.

14 Aujourd'hui, la taille moyenne des exploitations n'excède pas 0,75 ha dans la Plaine du Cul-de-Sac ou la plaine de Léogâne. Ces contrées comptent parmi les premières mises en 
culture par les colons français au XVII siècle et ce sont elles qui sont également les plus affectées par le séisme actuel. Comprendre l'ampleur de la catastrophe c'est admettre que les bidonvilles ne sont pas l'expression d'une forme particulière de tradition, ni une fatalité du paysage, mais le résultat d'une absence de considération publique d'un problème social. La reconstruction de la capitale haïtienne nécessite une refonte totale de l'aménagement urbain, guidée par l'urgence de reloger en toute sécurité le plus grand nombre, mais inspirée par la volonté de renforcer, en même temps que les murs, ébranlés par le séisme, les liens sociaux mis à mal par deux siècles de décadence économique et de marronnage social. 